\title{
Targeting the target of rapamycin (TOR): looking to mother nature
}

\author{
Eric K. Rowinsky
}

Received: 1 March 2011 / Accepted: 3 March 2011 /Published online: 27 April 2011

(C) Springer-Verlag 2011

Following the identification of a unique target for therapeutic development in cancer and other diseases, armies of medicinal chemists toil to identify novel active compounds, or "hits", which are typically found by screening compound libraries for the desired biological properties [1,2]. While there are a number of approaches to identifying hits, the most successful efforts have been largely due to the intuition of talented chemists gained through years of rigorous training and experience. Following the identification of therapeutic candidates, if any, the next step in drug discovery involves another army of chemists who seek to improve the biological and physiochemical properties of the hit $[3,4]$. Structural modifications are made to improve the recognition and binding geometries of the candidate compounds, their affinities and pharmacologic characteristics, and/or their reactivity and stability against metabolic degradation. While a sizeable proportion of efforts towards hit identification and lead optimization fail to result in therapeutic candidates, still others turn out reasonable clinical candidates with requisite specificity and potency, albeit generally in the micromolar, and occasionally, in the nanomolar range at best. However, compared to nature, with its processes of natural selection and evolution, armies of pharmaceutical chemists seem like mere amateurs. Not only do plants, fungi, and animals synthesize some of the most potent and specific small molecules, but natural selection and evolution have enabled living organisms to identify the best

\section{E. K. Rowinsky $(\square)$}

Primrose Therapeutics Inc.,

5 Robin Road,

Warren, NJ 07059, USA

e-mail: erowinsky@oncodrugs.com
“Achilles' heels" or targets to thwart [5-8]. Nature gave oncologists its targets and natural product anticancer agents in the early days of cancer medicine (e.g., microtubules/vinca alkaloids and taxanes; DNA/antitumor antibiotics; trabectedin/ toposiomerases/epipodyllotoxin and camptothecin, just to name a few), and nature just keeps on giving.

More than 30 years ago, a soil sample from Easter Island was found to contain a bacterial strain Streptomyces hygroscopius that produced a potent antifungal metabolite, later named rapamycin [8]. In addition, to its antifungal properties, rapamycin was subsequently found to possess potent antiproliferative and immunosuppressive properties, the latter of which lead to its approval for use as an immunosuppressant by the regulatory authorities in the United States and worldwide [9]. As has been common in discovery of biologically active natural products, but seemingly backward, first comes the discovery of the natural product itself. In a somewhat convoluted way, investigations into the mechanism of action of natural products are then followed by the discovery of its target, as well advancements in the understanding of normal biological and aberrant processes, like cancer. In essence, hits identified through random natural product screening have not only yielded new classes of therapeutics, but new information about normal and aberrant biology, as well as novel therapeutic targets.

The intracellular target of rapamycin, which was cleverly named the target of rapamycin (TOR), is a master integrator of cell energy state, nutrient status, and growth factor stimulation $[9,10]$. The kinase component of TOR has two distinct complexes, TORC1 and TORC2, and the network that regulates these two complexes is interconnected with distinct 
and overlapping inputs and outputs [9-12]. TOR-mediated and TOR-dependent processes are integrally involved in the genesis and maintenance of cancer, metabolic disorders, angiogenesis, and cardiac hypertrophy, as well as memory and learning. The articles published in Targeted Oncology's special focus on mTOR review the therapeutic aspects of targeting a wide array of cancers with therapeutics targeting TOR. These articles illustrate how TOR signaling is, in essence, an "Achilles' heel" for therapeutic development against cancer due to its central role in regulating cell growth, protein translation, autophagy, and metabolism. This two-part series focuses on how new pharmacological tools and their abilities to knockdown the function of complex-specific TOR subunits are providing new insights into their regulation and function in physiological contexts, as well as generating new avenues for therapeutic intervention in cancers associated with aberrant activity of cellular constituents. Understanding TOR has also furthered an understanding of the connectivity between cancer and aging, as reviewed by Sharp et al. [13] as well as the linkages between inflammation, stress, and cancer, as reviewed by Cam and Houghton [14], particularly with reference to hypoxic stress and the genesis of various pediatric malignancies.

The complexes that constitute TOR, TORC1 and TORC2, serve to integrate signals from several growth factors, largely amalgamating information about cellular energy and nutrient status [9]. Although both complexes are activated by receptor tyrosine kinases coupled to the phosphoinositide 3-kinase (PI3K) pathway, TORC1 is principally responsible for responding to signals related to cellular nutrient and energy status [9]. The kinase Akt, which is downstream of PI3K, activates TORC1, whereas Akt is also activated by TORC2 $[10,12]$. Because of the connectivity between TOR and the PI3K/Akt pathway, the components of which are frequently mutated in cancer, and since genes encoding components of the TORC1 pathway are also implicated in cancer, there is great interest in targeting this pathway therapeutically. With new pharmacological tools and the ability to selectively inhibit specific TOR complexes, the functions and regulation of TOR signaling are being progressively comprehended, which is leading to improved and effective therapies for diseases associated with aberrant signaling by TOR. For example, this understanding which, in part, encompasses the comprehension of the mechanisms of resistance of TOR inhibitors, as discussed by Carew et al. has heralded a new class of small molecules capable of targeting and robustly inhibiting both TORC 1 and TORC2 (TORC1/2), as discussed by Ogita and LoRusso [1517]. In contrast to rapamycin and rapalogs, TORC $1 / 2$ inhibitors have the potential of effectively target the PI3K/ Akt pathway, which not only remains activated, but is enhanced in a compensatory manner, following solitary targeting of TORC1 $[15,16]$.
Aberrant activation of a number of signaling moieties, both upstream and downstream of TOR, can drive TOR, and, in turn, also drive its primary outputs that are generally proliferative and anabolic. TOR can be activated secondary to the overexpression or activation of growth factor receptors, including human epidermal growth factor receptor 2 (HER-2) and insulin-like growth factor receptor, as well as amplifications/mutations in PI3K and Akt [18-21]. Furthermore, activating mutations of almost any downstream component of the mitogen-activated kinase pathway (MAPK), particularly RAF and RAS, can increase signaling through PI3K, thereby activating TOR $[9,22]$. Another, and perhaps the most important, mechanism responsible for the activation of PI3K, Akt, and/or TOR, especially in cancer, involves deletions of the tumor suppressor phosphatase and tensin homolog (PTEN), which is a negative regulator of PI3K signaling [23]. Suppression of PTEN is one of the most common aberrations found in cancer, including malignancies of the breast, endometrium, thyroid, brain and prostate, and melanoma; PTEN downregulation may be due to loss of heterozygosity, methylation, aberrant expression of regulatory microRNA, or protein instability [10, 23-27]. Perhaps the most significant clue that TOR targeting could impart robust therapeutic activity in a wide range of advanced cancers is that activated TOR signaling is associated with several tumor-predisposition syndromes such as Cowden's syndrome (caused by a PTEN mutation), PeutzJeghers syndrome (caused by an LKB1 mutation), tuberous sclerosis (caused by a TSC1/2 mutation), and neurofibromatosis (caused by a NF1 mutations) [10, 23-27].

In this special focus, Ogita and LoRusso describe the pursuit of mTOR-targeted inhibitors as chemotherapeutic agents, starting with rapamycin and rapalogs, which predominantly inhibit TORC1, newer agents that target the catalytic sites of both TORC1 and TORC2, and even newer generations of inhibitors of TOR and related pathways (PI3K, Akt, PDK-1) [15], while Soefje et al. review the toxicity profiles of these agents [28]. The two-part series also highlights the clinical results achieved with TOR inhibitors in patients with a variety of advanced cancers to date including: renal cancer as reviewed by Kumar Pal and Figlin [29], hematopoietic and lymphoid malignancies as reviewed by Kelly et al. [30], soft tissue sarcoma as reviewed by Vemulapalli et al. [31], pediatric malignancies as reviewed by Cam and Houghton [14] and various other relevant tumors/malignancies, including subependymal giant-cell tumors associated with tuberous sclerosis and neuroendocrine carcinoma as reviewed by Alvarado et al. [32].

Despite the irrefutable clinical benefit observed with rapalogs in the aforementioned adult and pediatric malignancies, largely as a result of randomized and rigorous clinical trials designed to detect clinically relevant differences in timeto-event endpoints, such as progression-free survival and 
overall survival, robust single-agent activity, as manifested by tumor regression and/or major impact on time-to-event endpoints, has been relatively low [10]. Therefore, it is imperative to identify predictive biomarkers to prospectively identify patients who will derive the most benefit from these agents. Furthermore, an understanding of susceptibility factors may facilitate the rational selection and development of combination therapies, ultimately enhancing the success of therapeutics targeting TOR. In this two-part special focus, Delbaldo et al. discuss the status of both susceptibility and resistance biomarkers, as well as the results of studies suggesting that mutations in KRAS, BRAF, and PI3K influence sensitivity to rapamycin analogs [33].

The discovery of the natural product rapamycin from a bacterium on Easter Island several decades ago has led to the identification of TOR and an understanding of its importance in signal transduction, cellular response to external stress, and overall control of cellular anabolism. This discovery has also unlocked its therapeutic potential in a several therapeutic areas pertaining to cancer, immunology, cardiovascular disease, inflammation, and aging. With regard to cancer, rapamycin, rapalogs, and newer classes of competitive TORC1/TORC inhibitors, as well as therapeutics that inhibit a wide variety of related cellular constituents are proving to be effective as anticancer agents in a broad range of advanced malignancies, but determining their optimal use (i.e., dose, schedule, ideal combinations, and enrichment strategies) will inevitably lead to more optimal targeting of this pathway for cancer therapy.

Billions of years of natural selection resulted in nature's choice of the target TOR to thwart, coupled with the design of rapamycin as its targeting therapeutic of choice for TOR, which has opened up new areas of research and therapeutic development. Perhaps one of the most important lessons learned is the potential consideration at reprioritizing natural product screening in our overall efforts to identify novel targets and therapeutics for cancer and other complex diseases.

Conflict of interest statement The author has no conflict of interest to declare.

\section{References}

1. Keseru GM, Makara GM (2006) Hit discovery and hit-to-lead approaches. Drug Discov Today 11:741-748

2. Ghosh S, Nie A, An J, Huang Z (2006) Structure-based virtual screening of chemical libraries for drug discovery. Curr Opin Chem Biol 10:194-202

3. Yang SY (2010) Pharmacophore modeling and applications in drug discovery: challenges and recent advances. Drug Discov Today 15:444-450
4. Andricopulo AD, Salum LB, Abraham DJ (2009) Structure-based drug design strategies in medicinal chemistry. Curr Top Med Chem 9:771-790

5. Molinski TF, Dalisay DS, Lievens SL, Saludes JP (2009) Drug development from marine natural products. Nat Rev Drug Discov 8:69-85

6. Harvey AL, Cree IA (2010) High-throughput screening of natural products for cancer therapy. Planta Med 76:1080-1086

7. Pfisterer PH, Wolber G, Efferth T, Rollinger JM, Stuppner H (2010) Natural products in structure-assisted design of molecular cancer therapeutics. Curr Pharm Des 16:1718-1741

8. Liu EH, Qi LW, Wu Q, Peng YB, Li P (2009) Anticancer agents derived from natural products. Mini Rev Med Chem 9:1547-1555

9. Guertin DA, Sabatini DM (2009) The pharmacology of mTOR inhibition. Sci Signal 2:1-6

10. Meric-Bernstam F, Gonzalez-Angulo AM (2009) Targeting the mTOR signaling network for cancer therapy. J Clin Oncol 27:2278-2287

11. Alessi DR, Pearce LR, García-Martínez JM (2009) New insights into mTOR signaling: mTORC2 and beyond. Sci Signal 2:1-4

12. Wullschleger S, Loewith R, Hall MN (2006) TOR signaling in growth and metabolism. Cell 124:471-484

13. Sharp D, Richardson A (2011) Aging and cancer: can mTOR inhibitors kill two birds with one drug? Targ Oncol. doi:10.1007/ s11523-011-0168-7

14. Cam H, Houghton PJ (2011) Regulation of mammalian target of rapamycin complex 1 (mTORC1) by hypoxia: causes and consequences. Targ Oncol. doi:10.1007/s11523-011-0173-x

15. Ogita S, LoRusso P (2011) Targeting phosphatidylinositol 3 kinase (PI3K)-Akt beyond rapalogs. Targ Oncol. doi:10.1007/ s11523-011-0176-7

16. Richard DJ, Verheijen JC, Zask A (2010) Recent advances in the development of selective, ATP-competitive inhibitors of mTOR. Curr Opin Drug Discov Devel 13:428-440

17. Carew JC, Kelly KR, Nawrocki ST (2011) Mechanisms of mTOR resistance in cancer therapy. Targ Oncol. doi:10.1007/s11523-011$0167-8$

18. Zhou BP, Hu MC, Miller SA et al (2000) HER-2/neu blocks tumor necrosis factor-induced apoptosis via the Akt/NF-kappaB pathway. J Biol Chem 275:8027-8031

19. Chung J, Bachelder RE, Lipscomb EA et al (2002) Integrin (alpha 6 beta 4) regulation of eIF-4E activity and VEGF translation: A survival mechanism for carcinoma cells. J Cell Biol 158:165-174

20. Cui X, Zhang P, Deng W et al (2003) Insulin-like growth factor-I inhibits progesterone receptor expression in breast cancer cells via the phosphatidylinositol 3-kinase/Akt/mammalian target of rapamycin pathway: Progesterone receptor as a potential indicator of growth factor activity in breast cancer. Mol Endocrinol 17: $575-588$

21. Stemke-Hale K, Gonzalez-Angulo AM, Lluch A et al (2008) An integrative genomic and proteomic analysis of PIK3CA, PTEN, and AKT mutations in breast cancer. Cancer Res 68:6084-6091

22. Lopez-Chavez A, Carter CA, Giaccone G (2009) The role of KRAS mutations in resistance to EGFR inhibition in the treatment of cancer. Curr Opin Investig Drugs 10:1305-1314

23. Bunney TD, Katan M (2010) Phosphoinositide signalling in cancer: beyond PI3K and PTEN. Nat Rev Cancer 10:342-352

24. Liaw D, Marsh DJ, Li J et al (1997) Germline mutations of the PTEN gene in Cowden disease, an inherited breast and thyroid cancer syndrome. Nat Genet 16:64-67

25. Shaw RJ, Bardeesy N, Manning BD et al (2004) The LKB1 tumor suppressor negatively regulates mTOR signaling. Cancer Cell 6:91-99

26. Inoki K, Zhu T, Guan KL (2003) TSC2 mediates cellular energy response to control cell growth and survival. Cell 115:577-590 
27. Johannessen CM, Reczek EE, James MF et al (2005) The NF1 tumor suppressor critically regulates TSC2 and mTOR. Proc Natl Acad Sci USA 102:8573-8578

28. Soefje SA, Karnad A, Brenner A (2011) Common toxicities of mammalian target of rapamycin inhibitors. Targ Oncol. doi:10.1007/ s11523-011-0174-9

29. Kumar Pal S, Figlin RA (2011) Future directions of mammalian target of rapamycin (mTOR) inhibitor therapy in renal cell carcinoma. Targ Oncol. doi:10.1007/s11523-011-0172-y

30. Kelly K et al (2011) Mammalian target of rapamycin as a target in hematological malignancies. Targ Oncol. doi:10.1007/s11523011-0175-8
31. Vemulapalli S, Alvarado Y, Sankhala K, Mita M, Mita A (2011) The role of mammalian target of rapamycin inhibitors in sarcomas. Targ Oncol doi:10.1007/s11523011-0179-4

32. Alvarado Y, Mita M, Vemulapalli S, Mahalingam D, Mita AC (2011) Clinical activity of mammalian target of rapamycin inhibitors in solid tumors. Targ Oncol doi:10.1007/s11523-0110172-y

33. Delbaldo C, Albert S, Dreyer C, Sablin MP, Serova M, Raymond E, Faivre S (2011) Predictive biomarkers for the activity of mammalian target of rapamycin inhibitors. Targ Oncol doi:10.1007/s11523-011-0177-6 